\title{
EMBARAZO Y ENFERMEDAD PULMONAR OBSTRUCTIVA CRÓNICA SEVERA: TODAVÍA UNA MUY RARA ASOCIACIÓN
}

\author{
Leonardo Sosa ${ }^{1}$, Mariela Téliz ${ }^{1}$, Claudia Solé ${ }^{1}$, Inés Alonzo ${ }^{2}$, Patricia Di Landro ${ }^{2}$, \\ Marta Gutiérrez ${ }^{3}$. \\ 1 Clínica Médica B, Departamento Clínico de Medicina; ${ }^{2}$ Clínica Ginecotocológica B, Departamento Clínico de Cirugía, \\ 3 Clínica Médica C, Departamento Clínico de Medicina; Hospital de Clínicas Dr Manuel Quintela, Facultad de Medicina, \\ Universidad de la República del Uruguay.
}

\section{RESUMEN}

Mujer de 37 años, multípara. Intensa fumadora, bronquítica crónica e historia de disnea sibilante de años de evolución. Cursando embarazo de 30 semanas de edad gestacional, mal controlado y mal tolerado por aumento progresivo de su disnea que llega a ser de mínimos esfuerzos en el último trimestre. Consulta por expectoración purulenta, fiebre e insuficiencia respiratoria. Es valorada con radiografía de tórax, gasometría y espirometría. Tratada en base a antibióticos, oxígeno y broncodilatadores. Se realiza inducción del parto a las 38 semanas. Requiere cesárea de urgencia por agravación de la insuficiencia respiratoria, obteniéndose recién nacido de $2688 \mathrm{~g}$ con Apgar 6 /9. La evolución de ambos fue favorable. Se discute la incidencia de esta rara asociación y su posible aumento en el futuro. Se revisan los aspectos espirométricos de mal pronóstico que podrían contraindicar la gestación y que permanecen controversiales.

\section{PALABRAS CLAVES: Embarazo, enfermedad pulmonar obstructiva crónica, EPOC, tabaquismo}

\section{SUMMARY}

Woman aged 37, multiparous. She was an intense smoker and has chronic bronchitis. She has sibilant dyspnea with years of evolution. Going throw pregnancy of 30 weeks of gestational age without the appropriate control and not well tolerated by their dyspnea. She consulted for purulent expectoration and fever. She was evaluated with thorax $\mathrm{x}$-ray, blood gas analysis and spirometry, and treated with antibiotics, oxygen and bronchodilators. Induction is performed at the 38th week of gestational age. It requires caesarean section for aggravation of the breathing inadequacy, being obtained a newborn of $2688 \mathrm{~g}$ with Apgar score 6/9. They both follow up favorably. We discuss the incidence of this strange association and it possible increase in the future. The spirometrycs aspects of bad prognosis are revised and that it might contraindicate the gestation, although remain controversial.

KEY WORDS: Pregnancy, chronic obstructive pulmonary disease, COPD, tabaquism 


\section{INTRODUCCIÓN}

Según el estudio "PLATINO" la enfermedad pulmonar obstructiva crónica (EPOC), si se toma como criterio diagnóstico un valor de $70 \%$ para la relación VEF1/CVF (relación fija), tiene una prevalencia en Uruguay de $19,7 \%$ y en caso de utilizar el criterio GOLD (Global Initiative for Chronic Obstructive Lung Disease) de 7,8\%. Cabe acotar que el estudio PLATINO excluyó menores de 40 años y también a embarazadas (1). Estas cifras coinciden en general con las informadas a nivel internacional, aunque hay una amplia variabilidad (2). Su mayor incidencia es sobre todo en hombres mayores de 45 años. Sin embargo, esta enfermedad viene experimentando profundos cambos epidemiológicos vinculados al género (3).

Llamativamente en el Reino Unido (RU) encuentran que la prevalencia en mujeres viene aumentando en las últimas décadas, particularmente en el grupo etario de 20 a 44 años y desde mediados de los 90' la enfermedad es más prevalente en mujeres (4). También la mortalidad por esta enfermedad está cambiando su perfil y tanto en Estados Unidos (EEUU) como en el RU se observa un aumento de la mortalidad en las mujeres y se estima que la diferencia con los hombres puede desaparecer en el futuro próximo $(4,5)$.

Todos estos cambios aún no han impactado en las mujeres en edad reproductiva y la ocurrencia de EPOC en ellas sigue siendo rara. A su vez, la experiencia publicada de la asociación de gestación y EPOC es escasa $(4,6)$. No obstante, debido al aumento de la incidencia del tabaquismo en las mujeres (7), su inicio más precoz en los últimos años (8), su probable mayor susceptibilidad en la adolescencia (9), la importancia que ha cobrado la observación de que la exposición al humo de biomasa en los países en vías de desarrollo afecta sobre todo a las mujeres (10), así como los cambios demográficos con respecto a la fecundidad que se están experimentando $(11,12)$, hace que la asociación de embarazo y EPOC pueda en el futuro no ser excepcional.

Por otra parte, los cambios fisiológicos que ocurren durante el embarazo y que repercuten en la mecánica ventilatoria, así como en los gases en sangre, hacen que la insuficiencia respiratoria durante la gestación suponga un riesgo considerable para el binomio materno fetal, a la vez que un desafío de manejo para el equipo tratante.

El objetivo de este trabajo es a propósito de un caso clínico, abordar y discutir varias interrogantes que se plantean acerca de la incidencia actual y futura de la asociación EPOC y embarazo, así como la determinación de ciertos parámetros de seguridad objetivos.

\section{Caso clínico}

Se trataba de una mujer de 37 años procedente de medio socioeconómico deficitario y con exposición a humo de biomasa durante al menos 12 años. Fumadora intensa desde la niñez, con un índice paquete/año de 42. Bronquítica crónica y portadora de disnea sibilante lentamente progresiva en los últimos 3 años. Gran multípara, cuyo último parto había sido 3 años antes por vía vaginal. Cursando gravidez no controlada de 32 semanas de edad gestacional. Desde hacía 2 meses tenía disnea de esfuerzo clase funcional $\|$ y 20 días previos al ingreso agudización de su sintomatología agregando expectoración purulenta y fiebre de hasta $39^{\circ} \mathrm{C}$. En el examen físico se destacaba: paciente lúcida, apirética, frecuencia respiratoria de 20 respiraciones por minuto y espiración prolongada. Fascie abotagada, con hiperemia conjuntival y presentaba dedos hipocráticos. A nivel pleuropulmonar la sonoridad global estaba aumentada y a la auscultación había gemidos y sibilancias difusas. En lo cardiovascular presentaba un ritmo regular 80 ciclos por minuto, no presentaba ingurgitación yugular ni edemas de miembros inferiores, la presión arterial era de 100/60 $\mathrm{mm} \mathrm{Hg}$.

La radiografía de tórax evidenciaba aumento del diámetro ápico-caudal, aplanamiento diafragmático, horizontalización costal e hiperclaridad pulmonar generalizada, no había imágenes de consolidación parenquimatosa (Figura 1). En la TAC de alta resolución de tórax se observaron áreas de enfisema centrolobulillar a predominio de ambos lóbulos superiores y segmentos apicales de los lóbulos inferiores, tractos densos a nivel hilioapical bilateral y enfisema paraseptal, no había otras alteraciones parenquimatosas (Figura 2).

Se realizó tratamiento en base a antitérmicos, oxígeno, antibióticos sistémicos (ampicilina sulbactam), broncodilatadores e hidrocortisona. En la evolución destacaba una gasometría, ventilando espontáneamente aire, que mostraba una insuficiencia respiratoria tipo II que mejoró con la oxigenoterapia (Tabla I). La espirometría evidenció alteración obstructiva muy severa, parcialmente reversible con broncodilatadores. El ecocardiograma transtorácico mostró cavidades normales, insuficiencia tricuspídea leve, sin evidencias ecocardiográficas de hipertensión pulmonar.

La determinación de alfa 1 antitripsina fue de $261 \mathrm{mg} / \mathrm{dL}$ (valor normal de 140 a $320 \mathrm{mg} / \mathrm{dL}$ ). El test del sudor realizado por método iontoforético y 


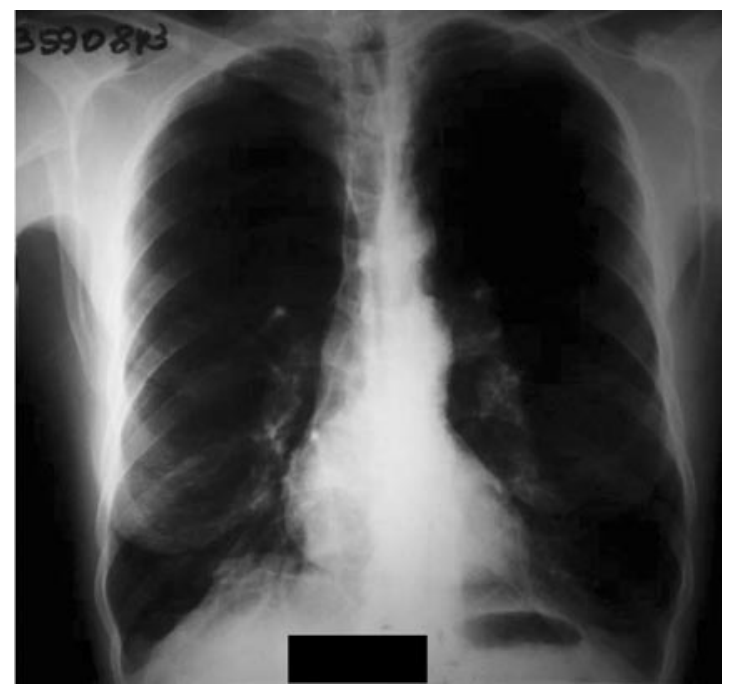

Figura 1. Caso clínico, radiografía de tórax (ver descripción en el texto).

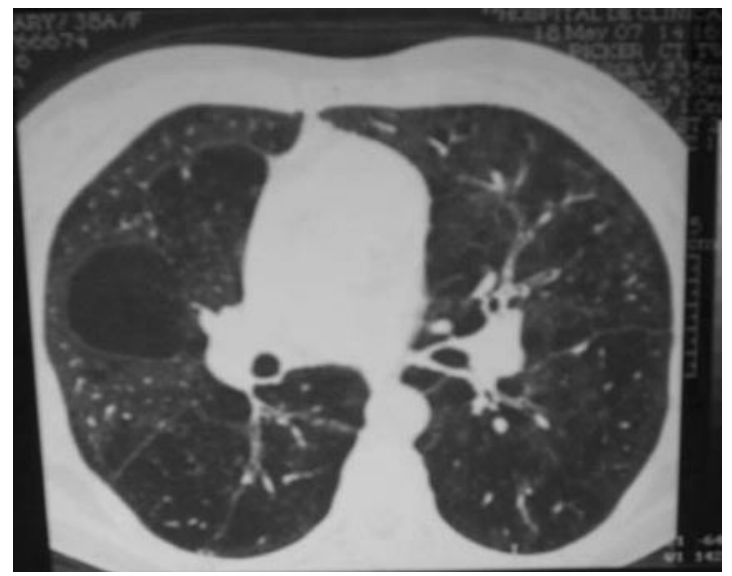

Figura 2. Caso clínico, tomografía de tórax de alta resolución (ver descripción en el texto). estimulación con pilocarpina fue negativo, con determinación de cloruro de $15 \mathrm{mEq} / \mathrm{L}$ para valores de referencia de hasta $50 \mathrm{mEq} / \mathrm{L}$.

A las 38 semanas de edad gestacional se decide interrupción del embarazo mediante inducción farmacológica con oxitocina. Previo al inicio se coloca catéter peridural para analgesia del trabajo de parto con el objetivo de disminuir el trabajo respiratorio generado por el dolor. La inducción se realiza durante 12 horas diarias por 3 días consecutivos. Durante la misma la paciente permaneció semisentada $y / 0$ en decúbito lateral izquierdo, con oxígeno a permanencia por cánula nasal y broncodilatadores. Se monitorizó con oxímetro de pulso. Se realizó además monitorización electrónica fetal continua, sin evidencia de sufrimiento fetal agudo. Al tercer día de inducción, presentó buena dinámica uterina, comenzando el trabajo de parto. En las siguientes 2 horas se evidencia aumento del trabajo respiratorio, por lo que se comienza ventilación no invasiva, no obteniéndose buena respuesta, lo que lleva a decidir realizar cesárea de urgencia (Tabla I). Se obtiene un recién nacido vivo de sexo femenino, Apgar 6/9, con un peso de 2688 g. Buena evolución puerperal con alta al quinto día en conjunto con neonato en buenas condiciones. Se realiza espirometría a los 2 meses del parto que no evidencia cambios significativos.

\section{DISCUSIÓN}

La asociación de EPOC vinculada al tabaquismo y embarazo es un hecho muy poco frecuente. Las pacientes embarazadas que presentan signos de insuficiencia respiratoria y obstrucción de la vía aérea que no son asmáticas deben ser estudiadas para descartar enfermedades crónicas como el déficit de alfa 1 antitripsina (a1AT), fibrosis quística 0 bronquiectasias.

Tabla I

GASOMETRÍAS ARTERIALES SERIADAS AL MOMENTO DE LA EVALUACIÓN

\begin{tabular}{llccccc}
\hline & $\mathrm{P}$ & $\mathrm{aO}_{2}$ & $\mathrm{PaCO}_{2}$ & $\mathrm{HCO}$ & $\mathrm{pH} \mathrm{B}$ & $\mathrm{E}$ \\
\hline Al ingreso (32 semanas) & $\mathrm{VEA}$ & 58,5 & 47,2 & 28,8 & 7,42 & 5,7 \\
Luego de tratamiento médico & $\mathrm{MFC} 28 \%$ & 97,5 & 46,5 & 24,8 & 7,34 & $-0,2$ \\
Inicio de inducción (38 semanas) & $\mathrm{CN}$ & 108,5 & 47,3 & 26,5 & 7,36 & 1,7 \\
Durante el trabajo de parto & CPAP & 122 & 49,1 & 22,4 & 7,28 & $-3,3$ \\
\hline
\end{tabular}

VEA: ventilando espontáneamente al aire. MFC: máscara de flujo controlado; CN: cánula nasal. CPAP: presión aérea positiva continua. $\mathrm{PO}_{2}$ : presión parcial de oxígeno. $\mathrm{PCO}_{2}$ : presión parcial de dióxido de carbono. $\mathrm{HCO}_{3}$ : bicarbonato; BE: exceso de base. 
La a1AT es una proteína capaz de interferir con varias enzimas proteolícas como la elastasa de los neutrófilos. Su déficit, una enfermedad hereditaria, se caracteriza por dificultad para interferir con estas proteasas. Característicamente cuando se asocia al tabaquismo produce enfisema pulmonar panacinar que puede manifestarse en edad reproductiva. Sin embargo la asociación con embarazo ha sido publicada sólo esporádicamente (13).

A su vez la fibrosis quística, otra enfermedad hereditaria, puede ser causa de EPOC severa. La expectativa de vida de las portadoras de esta enfermedad ha aumentado rápidamente en las últimas décadas. Actualmente en EEUU es de 31,6 años. Esto hace que no sea infrecuente que mujeres con esta enfermedad se embaracen. Tendrían los mismos resultados obstétricos que las mujeres sin fibrosis quística. Incluso los trabajos coinciden en mostrar mejor función pulmonar en las pacientes que se embarazan que en las que no lo hacen, probablemente merced a los cuidados que se les dispensan $(14,15)$.

La enfermedad bronquiectásica no ha sido informada particularmente en asociación con el embarazo. Su manejo terapéutico no debería diferir del de las no gestantes, excepción hecha del uso de aquellos antibióticos que sean riesgosos para el embrión o el feto.

Con respeto a la más frecuente de las enfermedades obstructivas como es el asma, es particularmente importante ser muy riguroso a la hora de diagnosticarla, ya que se ha demostrado que con las mujeres existe cierto sesgo a diagnosticar asma antes que EPOC (16). Por lo tanto se deberá tener alto índice de sospecha de EPOC ya que esta patología viene en aumento entre las mujeres y podría estar siendo subdiagnosticada.

$\mathrm{Si}$ bien la EPOC ha sido tradicionalmente mucho más frecuente en hombres mayores de 45 años, la tendencia actual podría cambiar esta situación. Se informan observaciones en EEUU y el RU de aumentos significativos en la prevalencia, tasas de internación y de mortalidad por EPOC entre las mujeres (4,5). En EEUU las hospitalizaciones por EPOC tienden a igualarse entre hombres y mujeres a partir del año 1989 y para 1998 son mayores las de las mujeres. Por primera vez en el año 2000, el número absoluto de mujeres muertas por EPOC es mayor que el de hombres (5). A su vez en el RU se ha observado que la prevalencia de mujeres, del grupo etario de 20 a 44 años con diagnóstico de EPOC, alcanza y supera la de hombres del mismo grupo etario hacia el año 1995 (4).

Estas tendencias podrían estar reflejando la mayor incidencia de tabaquismo en la mujer a partir de la década del 40' $(5,7)$. Incluso este aumento ha sido tan sostenido que actualmente hay países que ya informan mayor prevalencia de tabaquismo en mujeres que en hombres y advierten debido a este fenómeno, sobre el aumento en el futuro próximo, de las enfermedades tabaco dependientes en ellas $(7,8,17)$.

Además, el inicio del tabaquismo se ha ido trasladando a edades más tempranas. Este fenómeno se observa en Uruguay y en la Región, donde las adolescentes tienen una prevalencia de tabaquismo incluso, más alta que la de los varones (8). Este hecho tiene la gravedad adicional de poder provocar retraso del desarrollo pulmonar en este grupo etario, a lo que se agrega que la adolescente sería más vulnerable que el varón (9) Al respecto, se ha demostrado que el impacto del tabaquismo en la mujer, en general, sería mayor que en el hombre. Las mujeres tendrían mayor hiperreactividad bronquial en parte por tener una vía aérea de menor calibre (18) y también se ha informado mayor susceptibilidad al deterioro funcional (19). Asimismo, no sólo se informan mayores tasas de hospitalización y mortalidad entre las mujeres con EPOC, sino que desarrollan la enfermedad más precozmente y tienen una expresión más severa, aún expuestas a la misma cantidad de humo de tabaco que los hombres (20).

Adicionalmente, en poblaciones carenciadas, sobre todo de países subdesarrollados, se debe agregar como agente etiológico de la EPOC el humo de biomasa. Su calidad de vida, mortalidad y características clínicas son similares a la EPOC provocada por el tabaquismo. Por razones socioculturales las mujeres se ven más expuestas a este agente y por lo tanto las afecta con mayor frecuencia (10). En Uruguay, se agregan los cambios demográficos que se vienen produciendo y que aumentan las posibilidades de embarazos en mujeres mayores de 30 años. Este fenómeno podría contribuir a que la observación de EPOC en la embarazada en el futuro, no sea una excepción.

Si bien en Uruguay la tasa global de fecundidad se mantiene en descenso desde 1975, hay una tendencia en los últimos 40 años, muy leve pero sostenida, a la recuperación y ascenso de la fecundidad específica de las mujeres mayores de 30 años (fecundidad tardía) (21). Esto probablemente refleje la paulatina incorporación al mercado laboral y profesional de las mujeres y que haría posponer la concepción. Un fenómeno similar se observa en los últimos años en EEUU. Aún así, para los grupos de edad mayores de 30 años, Uruguay tiene tasas de fecundidad más altas que ese país (22).

La baja incidencia de embarazadas con insu- 
ficiencia respiratoria obstructiva crónica severa ha hecho que no haya pautas de manejo de este tipo de pacientes. Las controversias se centran en aquellos parámetros espirométricos y/o gasométricos que pueden predecir complicaciones y que podrían contraindicar el embarazo. Algunos autores han planteado por ejemplo, que un VEF1 inferior al $50 \%$ del predicto debería contraindicar el embarazo $(14,23)$. Reconociendo la limitación que supone la experiencia individual, otros autores proponen un valor de corte de VEF1 de 0,8 L a partir del cual se pueden esperar dificultades en el manejo respiratorio (6). La paciente que presentamos tenía un VEF1 de $0,51 \mathrm{~L}$, que correspondía a $20 \%$ del predicto, muy por debajo de las cifras informadas en casos aislados y que se podrían considerar de seguridad mínimas. Parece claro que se requiere mayor experiencia al respecto.

En referencia a los objetivos gasométricos, el margen podría ser más estrecho. La oxigenación fetal adecuada requeriría una $\mathrm{SaO} 2$ materna de aproximadamente $95 \%$ y una $\mathrm{PaO} 2$ de $70 \mathrm{~mm} \mathrm{Hg}$. No estaría tan definido el nivel de $\mathrm{PCO} 2$, pero sería deseable un nivel igual 0 menor de $45 \mathrm{~mm} \mathrm{Hg}$ de este parámetro (24).

\section{CONCLUSIONES}

La EPOC en mujeres en edad reproductiva sigue siendo una enfermedad rara y por lo tanto son escasas las comunicaciones de su asociación con el embarazo. En los próximos años esta enfermedad puede aumentar su incidencia debido al inicio más temprano del tabaquismo en las mujeres, así como al aumento de las gestantes añosas. Los valores espirométricos límites para contraindicar una gestación no están resueltos. El embarazo y el buen resultado obstétrico son posibles, pero es recomendable programarlo y considerar el tratamiento agresivo con unos objetivos gasométricos y/o pulsiométricos ambiciosos. Es necesaria mayor experiencia para poder realizar recomendaciones más específicas.

\section{BIBLIOGRAFÍA}

1. Muiño A, López Varela M, Menezes A. Prevalencia de la enfermedad pulmonar obstructivacrónica y sus principales factores de riesgo: proyecto PLATINO en Montevideo. Rev Med Uruguay 2005;21(1):37-48.

2. Buist AS, McBurnie MA, Vollmer WM, et al. International variation in the prevalence of COPD (the BOLD Study). Lancet 2007;370(9589):741-50.

3. Pederson AP, Hoyak KA, Mills S, Camp PG. Reflecting the changing face of chronic obstructive pulmonary disease. Sex and gender in public education mate- rials on COPD. Proc Am Thorac Soc 2007;4:683-5.

4. Devereux G. ABC of chronic obstructive pulmonary disease. Definition, epidemiology, and risk factors. BMJ 2006;332:1142-4.

5. Mannino DM, Homa DM, Akinbami LJ, Ford ES, Redd SC. Chronic obstructive pulmonary disease surveillance - United States, 1971-2000. MMWR Surveill Summ 2002;51:1-16.

6. Lalli CM, Raju L. Pregnancy and chronic obstructive pulmonary disease Chest 1981;80(6):759-61.

7. Joossens L, Sasco A, Salvador T, Villalba JR. Las mujeres y el tabaco en la Unión Europea. Rev Esp Salud Pública 1999;73(1):3-11.

8. Warren CW, Jones NR, Peruga A, et al. Centers for Disease Control and Prevention (CDC). Global youth tobacco surveillance, 2000-2007. MMWR Surveill Summ 2008:57(1):1-28.

9. Gold DR, Wang X, Wypij D, Speizer FE, Ware JH, Dockery DW. Effects of cigarette smoking on lung function in adolescent boys and girls. $\mathrm{N}$ Engl $\mathrm{J}$ Med 1996;335(13):931-7.

10. Ramírez-Venegas A, Sansores RH, Pérez-Padilla R, et al. Survival of patients with chronic obstructive pulmonary disease due to biomass smoke and tobacco. Am J Respir Crit Care Med 2006;173:393-7.

11. Damonte AM. Instituto Nacional de Estadísticas. Componentes del cambio poblacional. Disponible en: http:// www.ine.gub.uy/biblioteca/Variables $\% 20$ siglo $\% 20 x x /$ varsxxf2.htm Consultado el 11/02/08.

12. Encuesta Nacional. Reproducción Biológica y Social de la Población Uruguaya. Una aproximación desde la perspectiva de género y generaciones. Disponible en: $\quad$ http://www.psico.edu.uy/academic/sexrep/PRESE NTACION\%2012\%20DE\%20JULIO\%20version\%201 2.7.pdf. Consultado el 11/02/08.

13. Dempsey OJ, Godden DJ, Martin PD, Danielian PJ. Severe $\alpha 1$-antitrypsin deficiency and pregnancy. Eur Respir J 1999;13:1492-4.

14. Pernaut J, Audra P, Mossan C, Gaucherand P. Mucoviscidose et grossesse. À propos d'un cas de grossesse gémellaire et revue de la littérature. J Gynecol Obstet Biol Reprod 2005;34(1):716-20.

15. McMullen AH, Pasta DJ, Frederick PD, et al. Impact of pregnancy on women with cystic fibrosis. Chest 2006;129:706-11.

16. Camp PG, Goring SM. Gender and the diagnosis, management, and surveillance of chronic obstructive pulmonary disease. Proc Am Thorac Soc 2007;4:68691.

17. Becoña $E$, Vázquez Fernández LA. Las mujeres y el tabaco: características ligadas al género. Rev Esp Salud Pública 2000;74(1):13-23.

18. Kanner RE, Connett JE, Altose MD, et al. Gender differences in airway hyperresponsiveness in smokers with mild COPD: the Lung Health Study. Am J Respir Crit Care Med 1994;150:956-61.

19. Chen Y, Horne SL, Dosman JA. Increased susceptibility to lung dysfunction in female smokers. Am Rev Respir Dis 1991;143:1224-30.

20. Prescott E, Bjerg AM, Andersen PK, Lange P, Vestbo J. Gender difference in smoking effects on lung function and risk of hospitalization for COPD: results from 
a Danish longitudinal population study. Eur Respir J 1997;10:822-7.

21. Varela Petito C. El reemplazo de la población en el Uruguay, un fenómeno ausente en la agenda estatal. En: la fecundidad en América Latina: ¿Transición o revolución? Santiago de Chile. CEPAL, 2004: 399420. Disponible en: http://www.eclac.cl/publicaciones/ xml/3/22713/LCL2097.pdf. Consultado el 29/03/08.

22. Martin JA, Hamilton BE, Sutton PD, et al. Centers for Disease Control and Prevention National Cen- ter for Health Statistics National Vital Statistics System. Births: final data for 2005. Natl Vital Stat Rep 2007;56(6):1-103.

23. Wise RA, Polito AJ, Krishnan V. Respiratory physiologic changes in pregnancy. Immunol Allergy Clin North Am 2006;26(1):1-12.

24. Cole DE, Taylor TA, McCullough DM, Shoff CT, Derdak S. Acute respiratory distress syndrome in pregnancy. Crit Care Med 2005;33(10 Suppl):S269-78. 